\title{
Burnout y prescripción de incapacidad laboral temporal
}

\author{
Work satisfaction and temporary sick leave \\ prescription in a sample of doctors inside a mutual \\ society of industrial accidents and occupational \\ diseases (matepss).
}

\author{
Dr. Ismael S. Diana Domínguez \\ Comunidades de Castilla La Mancha \\ Correspondencia: \\ Dr. Ismael S. Diana Domínguez. Médico del Trabajo \\ Junta de Comunidades de Castilla la Mancha \\ Paseo de la Cuba, n'-27 \\ 02071 Albacete. España \\ Teléfono: 967195714 \\ Email: isdiana@jccm.es
}

Médico del Trabajo. S ${ }^{\circ}$ de Prevención de Riesgos Laborales de la Junta de

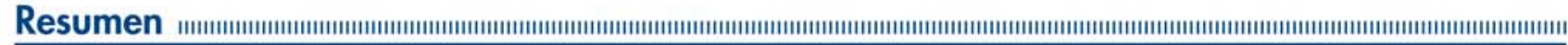

Objetivo: Hallar el grado de satisfacción laboral en una muestra de médicos asistenciales de una mutua de accidentes de trabajo y enfermedades profesionales (MATEPSS), determinando su relación con una serie de aspectos sociodemográficos y laborales y con su repercusión sobre la prescripción de incapacidad temporal.

Material y método: Se realizó un estudio transversal en una muestra final de 156 médicos (muestra total de 250 médicos), repartidos por toda la geografía española de una MATEPSS, a los que se les aplicó el Maslach burnout inventory, y un cuestionario de elaboración propia que mide una serie de variables sociodemográficas. Sobre 131 médicos pertenecientes a la muestra mencionada anteriormente, se realizó un estudio sobre la influencia que el burnout y esas variables sociodemográficas podían ejercer sobre la prescripción de bajas laborales. Se utilizó el paquete estadístico SPSS, realizándose dos métodos estadísticos diferentes.

Resultados: El primer método arrojó los siguientes resultados: la oportunidad de padecer grado alto de cansancio emocional se asocia de forma significativa $(\mathrm{p}<0,05)$, con padecer la sensación de carencia de material y de tiempo, no sentirse feliz con su trabajo, desear cambiarse de profesión y con el sentimiento de que los problemas laborales afectaban a su vida personal. El grado alto de despersonalización, con la sensación de que faltaba material para el trabajo diario, no sentirse feliz con su labor y sentir la intromisión habitual de los superiores en su trabajo, por otro lado, aparecieron como factores protectores contra la despersonalización, el ser mujer y trabajar exclusivamente en la mutua. La falta de realización personal aparece significativamente asociada con el pensamiento de no estar cumpliendo con sus expectativas profesionales, con creer que falta formación para que el personal desarrolle sus funciones correctamente, con desear cambiar de empresa y de profesión y con sentir la intromisión habitual de los superiores en su trabajo. Con respecto al estudio de las variables relacionadas con la hiperprescripción de bajas laborales llevado a cabo en la muestra de 131 médicos mencionada anteriormente, se asocia significativamente a la carencia de tiempo y de medios humanos, pero si realizamos el estudio por un segundo método estadístico, también aparece relación con la variable "intromisión habitual del jefe en su labor" aunque éste resultará ser un factor de protección, y desaparece la variable "sentir carencia de medios humanos". 
Conclusiones: el perfil del médico asistencial de mutua que padece burnout es el de un varón, pluriempleado, que no se siente feliz con su trabajo, y de hecho, cree que no está cumpliendo con sus expectativas profesionales. Los problemas laborales influyen en su vida personal. Considera que existe falta de material y tiempo para concluir sus tareas diarias y que el personal no está lo suficientemente bien formado para el desempeño de sus funciones, siente una intromisión habitual de sus superiores en su labor habitual y desea cambiar de empresa e incluso de profesión. Además, se trata de un médico especialista en medicina de familia.

El perfil del hiperprescriptor de bajas laborales es el de un médico, con independencia del sexo al que pertenece, y de si padece o no burnout, siente falta de tiempo para terminar su trabajo diario, falta de medios materiales y siente una frecuente intromisión de sus superiores en su labor habitual lo que le lleva a firmar un menor número de bajas laborales.

(Med Segur Trab (Internet) 2009; 55 (215): 72-81)

Palabras clave: burnout. Médicos asistenciales. Mutua. Incapacidad temporal.

Abstract

Objetive: Find the level of work satisfaction in a sample of doctors inside a mutual society of industrial accidents and occupational diseases (MATEPSS). In this report, the relation between work satisfaction, sociodemographics and industrial factors, and its influence on temporary sick leave is shown.

Teaching aid and method: A transverse study has been carried out on a final sample of 156 doctors (whole sample: 250 doctors) belonging to MATEPSS. The doctors are distributed in all Spanish geography. "The Maslach burnout inventory" was given to them. This questionnaire measures the burnout in its three sections: emotional tiredness, depersonalization and personal accomplishment. Another questionnaire was given also to them. This one, elaborated by the author, measures socio-demographics variables. On 131 doctors of the previous sample, it was made a study about the influence of burnout and socio-demographics variables on sick-leave prescriptions. Statistics-packet SPSS was used. Two different statistics methods were carried out in order to compare our results. First method (method 1 from now on) consists of the calculation of a " $\Psi^{2}$ " between all the variables had and, from this point, the calculation of a logistic binary regression between the variables that have emerged significant. Second statistic method (method 2 from now on) consists of the calculation of a logistic regression step by step conditioned to the significance coefficient.

Results: Method 1 showed that the possibility of suffer high level of emotional tiredness is connected with feeling of lack of resources and time with statistical significance $(p<0,05)$. Unhappiness with the job, wishes of a professional change, and the felling that personal life is affected by working problems are also connected with it in the same proportion. The high level of depersonalization resulted connected with the feeling of lack of resources, unhappiness with the work carried out and the feeling of a frequent interference in the work by a superior. On the other hand, protective-factors against depersonalization are being woman and working exclusively inside of mutual society. The absence of personal accomplishment resulted linked with statistical significance to the feeling of non-fulfillment with professional aspirations and the thought that workers do not develop correctly their duties due to a lack of training. Wishes of company change and the frequent interference in the work by a superior are also linked to it in the same ratio. In relation to factors connected with hyper-prescription of sick-leaves with method 1 it can be concluded they are connected with statistical significance to the lack of time and human resources. With method 2, the results are different. In this case, hyper-prescription appears also linked to variable "interference in the work by a superior" and the variable "lack of human-resource feeling" disappears.

Conclusion: The profile of a mutual society doctor who suffers burnout is: a man, with more than one work, unhappy with his job and with the believe that he is not achieving his professional aspirations. Working-problems affect his personal-life. He thinks he needs more resources and time to finish his tasks. He also thinks workers are not enough good-trained to develop correctly their duties. He feels he suffers a frequent interference in his work by a superior and he wishes a change of company and even his profession.

With a logistic regression step by step conditioned to the significance coefficient, it should be added that he is a family-doctor. 
The profile of hyper-prescriptor of sick-leaves is a doctor, man or woman with burnout or without it, who feels lack of time to finish the work, lack of resources and, considering method 2, with the feeling of a frequent interference in his/her job by a superior. This fact produces he/she signs a lower numbers of sick-leaves.

(Med Segur Trab (Internet) 2009; 55 (215): 72-81)

Key words: Burnout. Doctors. Mutual society. Temporary sick-leave. 


\section{INTRODUCCIÓN}

La incapacidad temporal se define como aquella situación en la que se halla un trabajador como consecuencia de una alteración de su estado de salud que precisa asistencia sanitaria y que le imposibilita temporalmente para trabajar. Estos trabajadores tendrán derecho a percibir un subsidio económico con el fin de paliar la ausencia de salarios derivada de la imposibilidad de trabajar. La gestión de la incapacidad temporal por contingencias comunes se produce en el ámbito de los servicios públicos de salud interviniendo en su gestión médica también los servicios médicos de la inspección sanitaria, de la inspección del INSS y de las propias mutuas; además no en el 100\% de los casos si la contingencia de origen es profesional, su gestión corresponde a las mutuas. Dicha gestión, ocasiona una serie de gastos económicos que deben ser controlados para el beneficio del país por ello decidimos que el objeto de este estudio sea la prevalencia del burnout en una muestra de médicos asistenciales de una M.A.T.E.P.S.S. su relación con una serie de variables sociodemográficas y laborales y si éstas y/o el burnout influyen sobre la prescripción de bajas laborales.

El concepto de burnout, conocido en España como síndrome de desgaste profesional ${ }^{1}$ o síndrome del quemado (término que se refiere a su traducción literal al castellano), fue descrito por primera vez por Freudenberg en 1974, siendo estudiado posteriormente por múltiples autores entre los que destacan Maslach y Jackson ${ }^{2,3}$, quienes lo definieron en tres dimensiones: El agotamiento o cansancio emocional (CE), que se refiere a la disminución y pérdida de recursos emocionales. La despersonalización (D) o deshumanización, que consiste en el desarrollo de actitudes negativas, de insensibilidad y de cinismo hacia los receptores de servicio prestado. La falta de realización personal (RP), que expresa las tendencias a evaluar el propio trabajo de forma negativa, con vivencias de insuficiencia profesional y baja autoestima personal. La asociación de este síndrome con una serie de variables sociodemográficas y laborales (edad, antigüedad en la profesión, sexo, etc.), algunas de las cuales han sido objeto de este estudio, plantea resultados contradictorios con los diferentes trabajos existentes en la literatura. De $\mathrm{Pablo}^{4}$ señala cuatro factores que influirían en la aparición del síndrome: La relación expectativas/realidad de lo que el profesional halla en su trabajo, el contexto socio-sanitario actual, el carácter particular del médico, y el factor organizativo de la empresa. En cuanto a las consecuencias, parece que existe una mayor unanimidad siendo las principales la pérdida de la calidad asistencial y el aumento del absentismo laboral.

\section{SUJETOS Y MÉTODO}

Se diseñó un estudio transversal observacional para conocer la prevalencia del burnout en los trabajadores de una M.A.T.E.P.S.S.. Esta mutua atiende a una población de más de 3,5 millones de trabajadores (año 2006). Tiene unos 1800 empleados, de ellos, en torno a 635 son médicos. Se remitieron 250 encuestas por todo el territorio nacional con el único criterio de que el entrevistador debía tener acceso al entrevistado mediante e-mail. Para acceder a dicha documentación se pidieron los permisos pertinentes y se aseguró la confidencialidad de los empleados.

De los 250 médicos a los que se les remitieron las encuestas, se eliminaron del estudio a aquellos que llevaban trabajando como médicos menos de tres años, aquellos que dijeron que no deseaban participar y aquellos que se dejaron más de dos ítems sin contestar, de este modo, quedo conformada la muestra por 156 médicos (muestra 1 de ahora en adelante), en quienes estudiaríamos el burnout y una serie de factores sociodemográficos.

De esta muestra 1, se extrajeron 131 médicos, es decir, el 84\% de la muestra 1, que denominamos como muestra 2 y en quienes se analizó la influencia del burnout y factores sociodemográficos sobre la prescripción de bajas laborales. Para ello, se determinó que 
serían hiperprescriptores, aquellos que estaban por encima del percentil 75 de bajas prescritas entre los años 2004 y primer semestre de 2006 (esto es, mayor o igual a 1076 bajas laborales prescritas).

Para el estudio del burnout se utilizó el Maslach Burnout Inventory (M.B.I) ${ }^{5}$, este cuestionario consta de 22 ítems con siete opciones de respuesta (escala de Likert de 0 a 6). Como comentábamos anteriormente, determina tres escalas que se obtienen sumando las puntuaciones de los ítems correspondientes. Las escalas de cansancio emocional y la de despersonalización, determinan un mayor grado de burnout si la puntuación es mayor mientras que la de realización personal refleja mayores niveles de burnout conforme la puntuación es menor. Los resultados obtenidos deben compararse con otros estudios para poder llegar a conclusiones ya que la puntuación de cada escala no es informativa por si sola. Para este estudio se ha escogido como puntos de corte el tercer cuartil para cansancio emocional y despersonalización situados en una puntuación de 28 y 12 respectivamente y el primer cuartil para la realización personal situado en 34. De esta forma se categorizaron las escalas en alto-no alto. Este sistema, seguido también por otros autores $^{7,6}$, es diferente a la escala propuesta por los autores del MBI, donde los puntos de corte coinciden con el tercer tercil. Como variables independientes se escogieron la edad, años de profesión como médico, los años de profesión en la mutua, trabajar exclusivamente en la mutua, el tipo de jornada laboral, tener la sensación de carencia de tiempo, material, medios humanos o formación, creer que existe habitualmente intromisión de los superiores jerárquicos en su labor, el deseo de cambiar de empresa o de profesión, el que los problemas laborales afecten a su vida extralaboral, el sentirse feliz con su trabajo, sentirse bien remunerado, estar cumpliendo con sus expectativas profesionales, trabajar habitualmente en un hospital o una delegación de la mutua, la especialidad de medicina que posee.

Análisis de los datos: El estudio estadístico se realizó con el paquete estadístico SPSS utilizando dos métodos distintos: El primer método ("método 1" de ahora en adelante), consistente en: primero, se realizó un estudio descriptivo de las variables recogidas. En segundo lugar, con objeto de hallar los factores que se encuentran asociados de manera independiente entre las diferentes variables realizamos un test de Chi cuadrado y a continuación, con aquellas variables que resultaron ser significativas $(\mathrm{p}<0,05)$, realizamos un estudio de regresión logística binaria siendo la variable dependiente cada una de las tres que se refieren al síndrome de burnout y las covariables las variables sociodemográficas. Cuando se obtenía un coeficiente de una variable con una significación mayor de 0.25 , ésta era eliminada del modelo obteniéndose una tabla que era la que finalmente se comentaba. En estos modelos se llevó a cabo el cálculo del test de bondad ajuste de Hosmer-Lemeshow que nunca dio significativo.

El segundo método estadístico ("método 2", de ahora en adelante), consistía en una regresión logística por pasos condicional al estadístico de significación.

\section{RESULTADOS}

Se cumplimentaron un total de 156 cuestionarios de los cuales se constata que 90 son hombres y 66 son mujeres, con una edad comprendida entre 28 y 62 años y una edad media de 41,59 años con una desviación típica de 6,42. Corresponden el mínimo y el máximo a 2 varones. En el caso de las mujeres, la más joven tendrá 30 años y la mayor 52. El médico de nuestro grupo que más tiempo lleva ejerciendo lo ha hecho durante 38 años. La media se sitúa en casi los 16 años y la mediana en los 15 con una desviación típica de 6,71 años. El resto de resultados obtenidos, los mostramos en la tabla 1. 
Tabla 1. Características sociodemográficas y laborales. N=156 médicos (muestra 1)

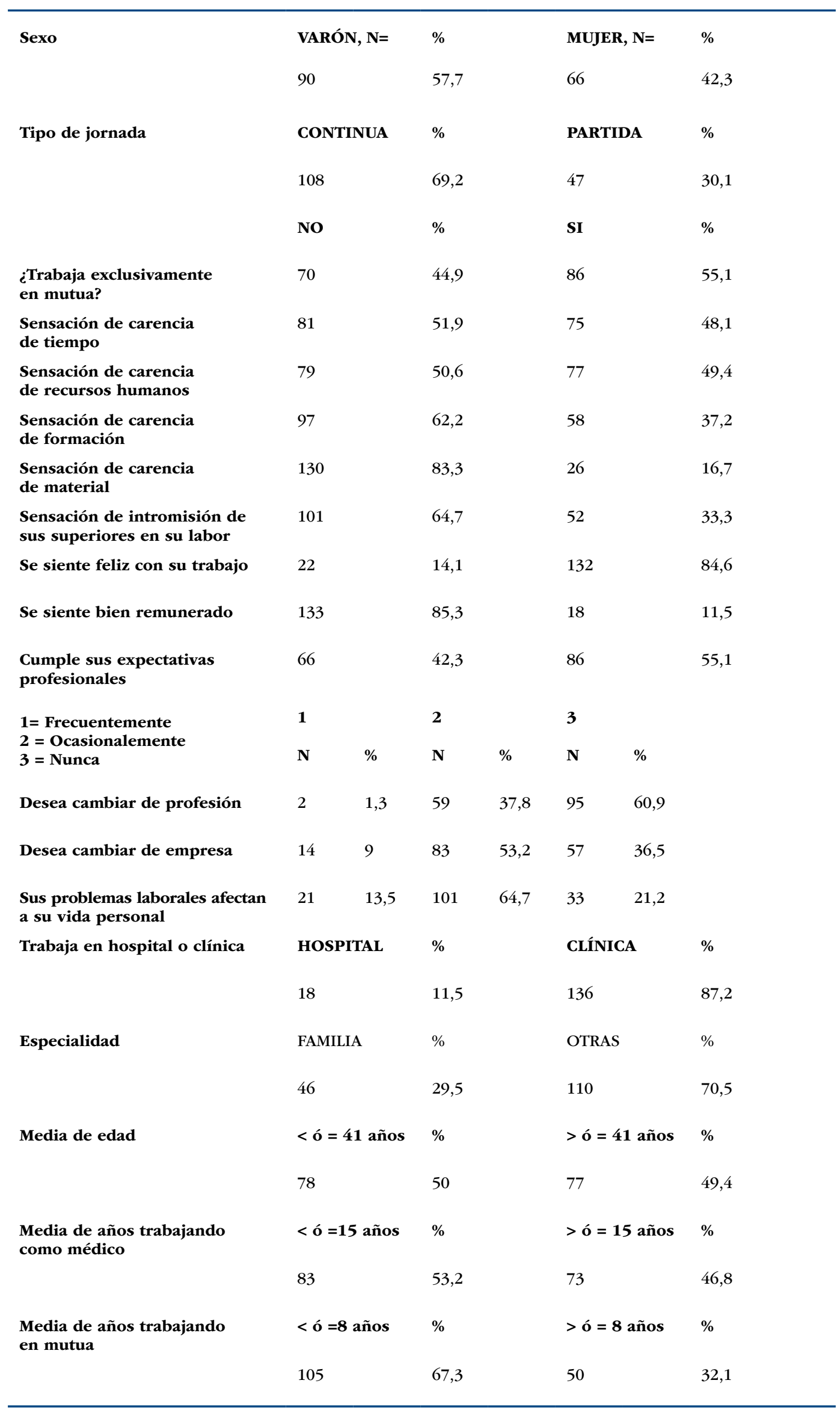


La tabla 2, muestra los niveles de desgaste profesional o burnout en cada una de sus tres escalas. Refiriéndonos a cada una de ellas por separado mostraron un alto grado de cansancio emocional 12 médicos, despersonalización, 14 médicos y únicamente falta de realización personal, 12 médicos. No presentaban niveles altos en ninguna de las tres escalas 83 médicos. Las posibles combinaciones entre las escalas fueron: Cansancio emocional y despersonalización a la vez: 25 médicos. Cansancio emocional y falta de realización personal: 17 médicos. Cansancio emocional, despersonalización y falta de realización personal: 13 médicos. Despersonalización y falta de realización personal: 19 médicos. Cansancio emocional y despersonalización pero no falta de realización personal: 12 médicos. Cansancio emocional y falta de realización personal pero no despersonalización: 4 médicos. Despersonalización y falta de realización personal pero no cansancio emocional: 6 médicos.

Tabla2. Grados de burnout en cada una de sus escalas y media para la muestra de 156 médicos (muestra 1)

\begin{tabular}{|c|c|c|c|c|c|c|}
\hline \multirow[b]{2}{*}{ GRADO } & \multicolumn{2}{|c|}{ Cansancio emocional } & \multicolumn{2}{|c|}{ Despersonalización } & \multicolumn{2}{|c|}{ Realización personal } \\
\hline & $\mathrm{N}$ & $\%$ & $\mathrm{~N}$ & $\%$ & $\mathrm{~N}$ & $\%$ \\
\hline BAJO & 39 & 25 & 31 & 19,9 & 35 & 22,4 \\
\hline MEDIO & 76 & 48,7 & 80 & 51,3 & 74 & 47,4 \\
\hline ALTO & 41 & 26,3 & 45 & 28,8 & 47 & 30,1 \\
\hline MEDIA & \multicolumn{2}{|c|}{22} & \multicolumn{2}{|c|}{8} & \multicolumn{2}{|c|}{40} \\
\hline
\end{tabular}

En la muestra formada por 156 médicos, y por el método "estadístico 1", el cansancio emocional alto se asoció significativamente $(\mathrm{p}<0,25)$, con la sensación de carencia de material, de formación y tiempo para terminar sus tareas, con no sentirse feliz en su trabajo, con desear cambiar de profesión y con que sus problemas laborales afecten habitualmente a su vida personal. El grado alto de despersonalización se asoció significativamente $(\mathrm{p}<0,05)$ con no sentirse feliz con su trabajo, con sentir que sus jefes se entrometen habitualmente en su labor y con el deseo de cambiar de empresa. Aparecen como factores protectores frente a la despersonalización, ser mujer y trabajar exclusivamente en la mutua. La falta de realización personal se asocia significativamente $(\mathrm{p}<0,05)$, con tener la sensación de que sus superiores se entrometen habitualmente en su labor, con el deseo frecuente de cambiar de empresa para la que trabaja e incluso de profesión, con el sentimiento de no estar cumpliendo con sus expectativas profesionales y con la sensación de carencia en la formación recibida para el desempeño de sus funciones. Con respecto a la muestra de 131 médicos ("muestra 2", perteneciente a la muestra 1), las variables que afectan al burnout son prácticamente las mismas y la hiperprescripción de bajas laborales, se asocia significativamente $(\mathrm{p}<0,05)$, a la sensación de padecer carencia de tiempo y de medios humanos. Cuando utilizamos una regresión logística binaria por pasos condicional al coeficiente de significación $(p<0,05)$ hacia delante, las variables que influyen en la hiperprescripción de bajas laborales son la sensación de carencia de tiempo y la sensación de que los jefes se entrometen habitualmente en sus tareas, si bien, este último factor actuaría como factor de protección.

Tabla 3. Niveles de burnout para cada escala y valor medio de cada una de ellas en la muestra estudiada de 131 médicos (muestra 2)

\begin{tabular}{|c|c|c|c|c|c|c|}
\hline \multirow[b]{2}{*}{ GRADO } & \multicolumn{2}{|c|}{ Cansancio emocional } & \multicolumn{2}{|c|}{ Despersonalización } & \multicolumn{2}{|c|}{ Realización personal } \\
\hline & $\mathrm{N}$ & $\%$ & $\mathrm{~N}$ & $\%$ & $\mathbf{N}$ & $\%$ \\
\hline BAJO & 35 & $26,7 \%$ & 44 & $33,6 \%$ & 34 & $26 \%$ \\
\hline MEDIO & 60 & $45,8 \%$ & 48 & $36,6 \%$ & 61 & $46,6 \%$ \\
\hline ALTO & 36 & $27,5 \%$ & 39 & $29,8 \%$ & 36 & $27,5 \%$ \\
\hline MEDIA & \multicolumn{2}{|c|}{22} & \multicolumn{2}{|c|}{8} & \multicolumn{2}{|c|}{39} \\
\hline
\end{tabular}


La tabla 3 presenta los niveles de burnout en la citada muestra 2 para sus tres dimensiones, podemos observar que apenas existen diferencias con la muestra 1 aunque ésta posee una media para la realización personal algo mayor.

\section{DISCUSIÓN}

Las variables sociodemográficas referidas a edad, sexo y años de vida laboral de nuestra muestra no difieren en gran medida de otros estudios similares realizados a otros grupos de profesionales del sector sanitario: ${ }^{21,10,11}$. Cabe destacar, que igual que en otros estudios $^{12,13}$, la distribución de cansancio emocional de grado alto y la falta de realización personal, encajan con la concepción de Chernis ${ }^{14}$ del burnout, quien habla de este síndrome como un fenómeno dinámico y evolutivo por el que son los años que corresponden a la edad media de la vida (rango de 41 a 45), los más susceptibles de padecerlo. En el caso de la despersonalización, apenas existe en nuestro estudio diferencia entre el rango 36 a 40 y 41 a 45, (rangos de edad y puntos de corte para burnout igual que en el estudio de Molina ${ }^{12}$ ). Tampoco encontramos unanimidad respecto a la influencia de la antigüedad profesional. Hay autores ${ }^{6,15,16}$, que mencionan la relación de niveles altos de burnout con la inexperiencia y falta de desarrollo de estrategias de afrontamiento para prevenir situaciones estresantes, o bien porque es en estos primeros años de carrera profesional el periodo en el que se produce la transición de las expectativas idealistas hacia la práctica cotidiana, teoría con la que no coincidimos según lo enunciado en el párrafo anterior, si bien es cierto que en nuestra muestra fueron excluidos aquellos profesionales con menos de tres años de ejercicio profesional.

En el gráfico 1 exponemos una comparación entre las medias obtenidas en nuestro estudio y las observadas en otros existentes en la bibliografía:

Gráfico 1. Comparación de diferentes estudios de burnout

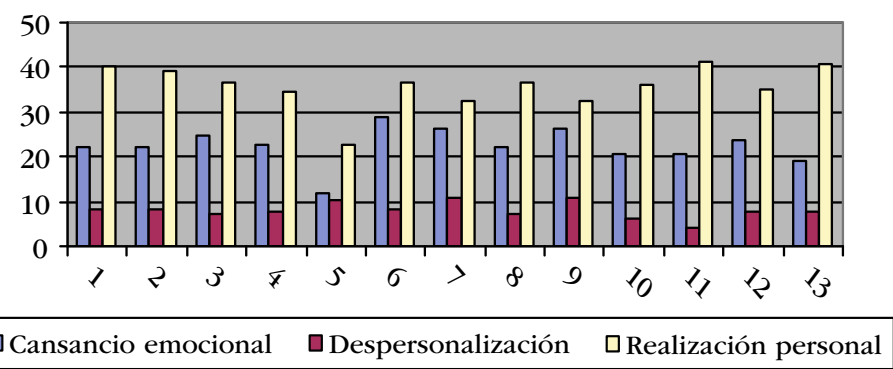

MUESTRA 1 ( médicos de mutua, $\mathrm{N}=156$ )

MUESTRA 2 (médicos de mutua, $\mathrm{N}=131$ )

TENERIFE (3) CUEVAS et al, [17]

CACERES (4) PRIETO el al, [15]

GUADALAJARA (5) ATANCE [16]

ZARAGOZA (6) de PABLO et al [18]

MASLACH MEDICOS ESPAÑOLES (7), [5]

8. MASLACH MEDICOS USA (8),[5]

9. MEDICOS DE ÁVILA (9)[6]

10. GIL-MONTES Y PEIRO (10)[6]

11. ODONTOLOGOS DE MURCIA (11),[6]

12. MEDICOS GERONA GRAU A. et al (12), [20]

13. MEDICOS DE MATARÓ, PERA G. et al(13),[19]

(La referencia de cada muestra corresponde al encuadrado en los paréntesis)

(El número que hay entre corchetes corresponde a la referencia bibliográfica)

Por lo tanto, podemos observar que nuestra muestra presenta unos resultados en las escalas cansancio emocional, despersonalización y realización personal pertenecientes al burnout relativamente buenos si tenemos en cuenta las medias presentadas en otros estudios.

Entre estos estudios de referencia, podemos observar las buenas puntuaciones obtenidas por los odontólogos de Murcia quienes pertenecen, igual que nuestra muestra, al ámbito de la empresa privada con las particularidades que ello tiene, además, en el caso del citado estudio, se suma la característica de que la mayoría de la muestra pertenece al régimen de autónomos con lo que verían una relación directa entre el esfuerzo que 
realizan en el trabajo y la compensación económica y social que reciben, variable esta última que se relaciona con el burnout según algunos estudios ${ }^{18}$.

En el estudio llevado a cabo por Escribá-Agüir y Bernabé-Muñoz ${ }^{22}$, se señalan como factores de riesgo psicosocial debido a la organización del trabajo, la sobrecarga de trabajo y la falta de personal, conclusiones a las que llega mediante un estudio de metodología cualitativa a partir de entrevistas de personal médico de seis hospitales públicos de la provincia de Valencia. Dichos resultados estarán en concordancia, por tanto, con los nuestros donde la sensación de carencia de tiempo para terminar su trabajo aparece relacionada con el cansancio emocional y con la hiperprescripción de bajas laborales. También la sensación de falta de medios humanos aparece relacionada con esta hiperprescripción.

Al observar las medias de estas escalas de las muestras de hiperprescriptores y normoprescriptores (tabla 4), pertenecientes a lo que hemos denominado muestra 2 ( $\mathrm{N}=131)$, vemos que los resultados continúan en la línea de lo que acabamos de comentar, aunque cabría señalar que el primero de estos dos grupos presenta una media superior en la escala de cansancio emocional y una media inferior en las escalas de realización personal y de despersonalización.

Tabla 4. Niveles de burnout para hiperprescriptores y normoprescriptores de bajas laborales en la muestra 2 ( $N=131$ médicos)

\begin{tabular}{lccc}
\hline & Cansancio emocional & Despersonalización & Realización personal \\
\hline HIPERPRECRIPTORES & 23 & 8 & 38 \\
NORMOPRESCRIPTORES & 22 & 9 & 40 \\
\hline
\end{tabular}

En nuestro estudio no hallamos relación estadísticamente significativa entre el síndrome de burnout y la prescripción de bajas laborales. Si encontramos relación estadísticamente significativa entre hiperprescripción de bajas laborales con las variables "sensación de carencia de tiempo" y "sensación de carencia de medios humanos". Sin embargo, cuando realizamos el estudio mediante el método de regresión logística binaria por pasos condicional al coeficiente de significación, no nos aparece la variable "sensación de carencia de medios humanos " pero si continúa apareciendo la variable "sensación de carencia de tiempo" y se introduce la variable "¿cree que su jefe se entromete habitualmente en su labor?", surgiendo la respuesta afirmativa a dicha cuestión como un factor de protección frente a dicha hiperprescripción de bajas. Por el contrario, resulta ser un factor de riesgo para la despersonalización. Así pues, esto nos podría estar confirmando la importancia de la figura de los superiores para el control del número de prescripciones de bajas laborales y por tanto para el control de la gestión de la incapacidad temporal.

\section{BIBLIOGRAFÍA}

1. Estudio del Síndrome de Desgaste profesional entre los médicos de un Hospital General”, Enrique Daniel Vega. Tesis doctoral. Fecha de lectura: 30/05/95. Universidad de Salamanca.

2. Instituto Nacional de Estadística- encuesta trimestral de coste laboral 2005.

3. "Las mutuas de accidente de trabajo y la prestación económica de incapacidad temporal por contingencia común". www.amat.es/documentos/prestacion\%20economica.pdf

4. Libro ponencias y comunicaciones del 16 Congreso de la Sociedad Valenciana de Medicina Familiar y Comunitaria. Importancia del Síndrome de Burnout, Factores determinantes y su prevención. Rafael de Pablo.

5. Maslach C, Jackson S.E. MBI. Inventario Burnout de Maslach. Síndrome del quemado por estrés laboral asistencial. Publicaciones de Psicología aplicada. Serie menor, núm.211.Madrid:TEA Ediciones. 1997.

6. Análisis de la ansiedad, burnout y otras manifestaciones de estrés laboral en los profesionales de la odontología de la región de Murcia. Maria Dolores Marín García.Tesis doctoral. Año 2005. 
7. J. Cebriá, J. Sobrequés, C. Rodríguez, J, Segurá. Influencia del desgaste profesional en el gasto farmaceútico de los médicos de atención primaria. Gace. Sanit. 2003;17(6):483-9

8. Ordenes D, Nadia. Prevalencia de Burnout en trabajadores del hospital Roberto del Río. Rev. chil. pediatr. [online]. oct. 2004, vol.75, no.5 [citado 31 Octubre 2007], p.449-454. Disponible en la World Wide Web: $<$ http://www.scielo.cl/scielo.php?script=sci_arttext\&pid=S0370-41062004000500006\&lng=es\&nrm=iso $>$. ISSN 0370-4106.)

10. http://dialnet.unirioja.es/servlet/articulo?codigo=723162: Estudio sobre la prevalencia del burnout en los médicos del Área Sanitaria de Talavera de la Reina. Autores: I. Bru Espino, A. Segura Fragoso, C. del Castillo Comas, E. Magaña Loarte, A. Franco Moreno, A. Martínez de la Casa Muñoz. Localización: Atención primaria: Publicación oficial de la Sociedad Española de Familia y Comunitaria, ISSN 0212-6567, Vol. 32, №. 6, 2003 , pags. 343-348

11. M. Esteva, C. Larraz, F. Jiménez. La salud mental en los médicos de familia: Efectos de la satisfacción y el estrés en el trabajo

12. A. Molina Siguero, M.A. García Pérez, M. Alonso González, P.Cecilia Cermeño. Prevalencia de desgaste profesional y psicomorbilidad en médicos de atención primaria de un área sanitaria de Madrid. Atención Primaria. 2003;31:564-571

13. Vol. 13 - Núm. 4- Abril 2003. MEDIFAM 2003; 13: 325-328.cartas al director

14. Cherniss C. Long-term consequences of burnout: An exploratory study. Journal of organizational behaviour 1992; 13(1): 1-11

15. L. Prieto Albino. Burnout en médicos de atención primaria. Lunes 15 Octubre 2001. Volumen 28 - Número 06 p. $444-445$. Atención primaria

16. J.C. Atance Martínez. Aspectos epidemiológicos del síndrome de burnout en personal sanitario. Rev.Esp. Salud Pública:1997;293-303. No -3, mayo-junio.

17. C. de las Cuevas, J.A. De la Fuente, M. Alviana, A.Ruiz-Benitez E. Coiduras-T.González-A.Moujir- M.T. Rodriguez. Desgaste profesional y clima laboral en atención primaria. Monografía

18. Prevalencia del síndrome de burnout o desgaste profesional en los médicos de atención primaria. $\mathrm{R}$ de Pablo González, JF Suberviola González. Fuente: Atención primaria. Lunes 30 Noviembre 1998. Vol. 22 Número 9 p. 580 - 584

19. Prevalencia del síndrome del quemado y estudio de los factores asociados en los trabajadores de un hospital comarcal. G Pera, M Serra-Prat .Gaceta sanitaria. 2002;16:480-6

20. A. Grau, R. Suñer, María M.García. Desgaste profesional en el personal sanitario y su relación con los factores personales y ambientales. Gaceta sanitaria. 2005; 19(6):463-70

21. Ordenes D, Nadia. Prevalencia de Burnout en trabajadores del hospital Roberto del Río. Rev. chil. pediatr. [online]. oct. 2004, vol.75, no.5 [citado 31 Octubre 2007], p.449-454. Disponible en la World Wide Web: $<$ http://www.scielo.cl/scielo.php?script=sci_arttext\&pid=S0370-41062004000500006\&lng=es\&nrm=iso $>$. ISSN 0370-4106.)

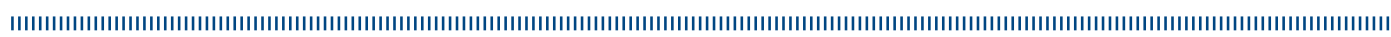

